\title{
Opportunities for Low-Cost Particulate Matter Sensors in Filter Emission Measurements
}

\author{
Almuth D. Schwarz ${ }^{1}$, Jörg Meyer ${ }^{1}$, Habil. Achim Dittler ${ }^{1}$ \\ ${ }^{1}$ Karlsruhe Institute of Technology, Institute of Mechanical Process Engineering and Mechanics, Straße am \\ Forum 8, 76131 Karlsruhe, Germany \\ *Correspondence: Almuth D. Schwarz (E-mail: almuth.schwarz@kit.edu), Karlsruhe Institute of Technology, \\ Institute of Mechanical Process Engineering and Mechanics, Straße am Forum 8, D-76131 Karlsruhe, Germany
}

\section{Abstract}

Commercial low-cost sensors for particulate matter measurements are a fast growing market and find many applications in the PM immission characterization in the environment. This application includes uncontrolled factors such as humidity, making the devices more prone to errors. These factors are controllable in closed emission control applications, turning these affordable sensors into potential monitoring devices for exhaust concentrations. In this study, time-resolved PM emissions of a surface filter were measured at various concentration levels. The suitability and limitations of a SDS011 PM2.5 low-cost sensor were evaluated by comparing its PM readings to a welas 2100 sensor by Palas ${ }^{\circledR}$. Experimental setup and results are presented and further discussed in this paper.

Keywords: SDS011 sensor, Emission monitoring, Low-Cost sensors, Particulate matter, measurements, Performance evaluation

\section{Introduction}

The application of low-cost particulate matter (PM) sensors is a fast growing area of research. It is long known that air pollution is an impediment to human health [1-5]. Respirable particles, i.e. particulate matter with an aerodynamic diameter of less than $2.5 \mu \mathrm{m}$, have especially been identified to cause health issues and lung cancer $[6,7]$. Traditionally, air quality sensors implemented by government authorities and scientists are complex and stationary, and require large economic investments, all of which making them inappropriate for applications where many devices are needed, such as air quality mapping. However, the increasing public concern about air quality and the need for high area coverage data recordings has lead to the rising development of low-cost, portable, personal PM sensors, introducing an attractive, affordable addition to conventional devices.

As a result, there is a rising public involvement in air quality studies. Citizen science projects worldwide such as EveryAware [8], Open Sense [9], Citi-sense (terminated) [10] or AirCasting [11] use low-cost sensor platforms to collect air quality data providing large area coverage of air quality measurements. The OK Lab Stuttgart is part of the 'Code for Germany' program by the Open

Received: April 27, 2018; revised: May 31, 2018; accepted: July 05, 2018

This article has been accepted for publication and undergone full peer review but has not been through the copyediting, typesetting, pagination and proofreading process, which may lead to differences between this version and the final Version of Record (VOR). This work is currently citable by using the Digital Object Identifier (DOI) given below. The final VoR will be published online in Early View as soon as possible and may be different to this Accepted Article as a result of editing. Readers should obtain the final VoR from the journal website shown below when it is published to ensure accuracy of information. The authors are responsible for the content of this Accepted Article.

To be cited as: Chem. Eng. Technol. 10.1002/ceat.201800209

Link to final VoR: https://doi.org/10.1002/ceat.201800209

This article is protected by copyright. All rights reserved. 
Knowledge Foundation Germany. The organization recently received a lot of public attention for its published PM data, which was measured with low-cost SDS011 sensors by Nova [12], reporting very high PM10 and PM2.5 values [13]. Because of its popularity, the same sensor model was subject of this study.

Although results of these citizen science projects record tendencies, the accuracy and precision of low-cost sensors is frequently questioned as no standardized implementation procedures are in place and precise manufacturing and calibration information are usually unavailable. Various types of micro sensors were tested aiming to evaluate their accuracy. Experiments were conducted as field studies or laboratory experiments or a combination of the two, employing professional devices as reference instruments. The majority of studies report mixed results of the low-cost sensors' performance in terms of precision and accuracy [14-20]. An attempt has been made to find correction factors in order to increase accuracy [14, 21-23]. Relative humidity has been identified as one of the main factors contributing to errors in measurement of low-cost PM sensors [19$21,23,24]$. The measured particles are to a certain degree hygroscopic and increase in size as they absorb water from their surroundings. As low-cost sensors measure the wet-size of the particles and are generally not able to differentiate between particles and small water aerosol droplets, rather than measuring dried particles like most reference devices with integrated heating equipment, this leads to great differences in results. Crilley and Sousan reported a general overestimation by lowcost PM sensors and noted that the smaller the measured particles were, the larger the associated error seemed to be $[16,21]$. Additionally, discrepancies were recorded between individual sensors of the same model, introducing a greater amount of uncertainty $[14,19]$. To minimize any errors, the appropriate and frequent calibration of low-cost sensors was suggested [16, 18, 21, 23]. Liu emphasized the importance of a steady state particle concentration during the calibration process [18].

The aim of this study is to explore a new field of applications for low-cost PM sensors. Their low economic investment of often 1000 times less than professional devices also makes them attractive for emission measurements in various research areas. Especially in cases where many individual sources contribute to one overall emission outlet, low-cost sensors could be the chance to record individual emission contributions and allow an instantaneous high spacial resolution. To make this possible a reasonable accuracy in results has to be confirmed. In previous laboratory studies measuring ambient air quality, low-cost PM sensors were found to provide reasonable precision provided proper calibration took place $[16,25]$. Many factors, which decrease the usability of results provided by low-cost sensors as reported for immission measurements in field studies, such as temperature and humidity, can be more easily monitored and controlled during emission measurements in laboratory settings. This could potentially make low-cost sensors comparable to verified professional devices during the emission monitoring in research and industrial settings.

In this paper a first evaluation on the possibilities and applications of low-cost PM sensors for the continuous measurement of emissions is presented. The goal is to introduce already existing sensors as an affordable alternative for distinct applications in research and industrial settings. Although current low-cost sensors are intended for immission measurements and would require a new calibration using particles relevant for the new target application, a first performance test of available low-cost sensors in emission measurements is presented in this paper.

In this study, an inexpensive NOVA SDS011 sensor, a low-cost PM sensor that is widely used for immission measurements, e.g. by the OK Lab Stuttgart [12], is tested in measuring the emissions of a filter. Results are compared with a professional, and highly reliable welas 2100 sensor by Palas ${ }^{\circ}$ and a traditional cyclone as time-averaging gravimetrical reference device. 


\section{Instrumentation and experimental methods}

\subsection{The SDS011 low-cost PM sensor}

Tab. 1 summarizes the specifications of the low-cost PM sensor and the reference instruments used in this study. The low-cost PM Optical Particle Counter (OPC) being subject of this study is the SDS011 manufactured by Nova [26]. The sensor can be purchased for a retail price of approx. $30 €$, depending on the vendor, making it roughly 1000 times cheaper than the reference OPC. The SDS011 officially records PM2.5 and promises to detect particles from $0.3 \mu \mathrm{m}$ in size up to a maximum concentration of $999.9 \mathrm{\mu g} \mathrm{m}^{-3}$ at maximum temperature and humidity of $50{ }^{\circ} \mathrm{C}$ and $70 \%$ in working environment, respectively. With a size of $71 \mathrm{~mm} \times 70 \mathrm{~mm} \times 23 \mathrm{~mm}$ and a weight of $45 \mathrm{~g}$, it is a light tool easily fitting on a palm. A maximum relative error $\pm 15 \%$ and $\pm 10 \mu \mathrm{g} \mathrm{m}^{-3}$ are specified by the manufacturer. It should be noted at this point, that next to the PM2.5 values, the sensor provides a second signal for PM10 values. This function is not promoted by the producer nor the vendor, but will be evaluated in this paper as well.

Table 1

Fig.1 shows a schematic diagram of the SDS011. It is based on optical light scattering principles. The gas enters the sensor through a circular opening and is channeled through a laser beam. When particles cross the beam, light is scattered and absorbed. The scattered light is detected in a $90^{\circ}$ angle by a photodetector placed at the bottom of the sensor. No further optical system in form of lenses is in place. The detected intensity of light corresponds to particle sizes according to the incorporated calibration curve. An unknown test dust is used to correlate light intensity and particle sizes. Further information about the calibration process is not available at this point. The output signal is in the form of pulse width modulation. The sensor is equipped with an integrated fan,

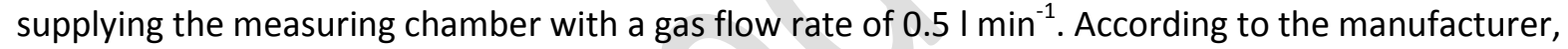
a supply tube of up to a maximum of $1 \mathrm{~m}$ length and $6 \mathrm{~mm}$ inner diameter can be used to connect the sensor to the sampling source [16]. In this experiment, it was decided to place the low-cost sensor directly in the measuring chamber without supply tube to minimize any uncertainties or errors in measurements.

Figure 1

\subsection{Reference instruments}

Two reference devices based on different measuring principles are used in this study. In this work, the reference devices are used to allow a qualitative and quantitative comparison to the results provided by the low-cost sensor. They are not intended for PM sensor calibration at this point.

\subsubsection{Promo 2000 with welas 2100}

The Promo 2000 manufactured by Palas ${ }^{\circ}$ can be purchased for approximately $30,000 €$. In this study it was used with a welas 2100 sensor [27]. Next to PM2.5 and PM10 data, a large range of other information about the particles is provided including particle size distribution, weight distributions, PM1, PM4 and total concentration curves. The Promo 2000 has a small, well-defined measuring volume, allowing to measure up to $10^{6}$ particles per $\mathrm{cm}^{3}$. It has the dimensions of $185 \mathrm{~mm} \times 450 \mathrm{~mm} \times 315 \mathrm{~mm}$ with an associated weight of ca. $8 \mathrm{~kg}$ with an extra $2.8 \mathrm{~kg}$ for the sensor, making it much less mobile than the SDS011 (Fig. 2). This OPC is also based on optical light scattering principles, but employs white light and is calibrated using monodisperse polystyrene particles of different sizes. Detected signals are categorized according to signal height and signal length, which correspond to particle size and speed, respectively. The categorization into multiple size classes (up to 128 classes per measuring range) makes this device much more precise than the SDS011. In this study it is set to a dust with a refractive index of 1.59 and a density of $1000 \mathrm{gl}^{-1}$. The Promo 2000 is equipped with an integrated pump, sampling $5 \mathrm{I} \mathrm{min}^{-1}$ of gas. The welas 2100 was 
chosen as reference device as it was tested in previous studies and is a proven reliable sensor model, well-known to the authors. In the following sections the combination of Promo ${ }^{\circ} 2000$ and welas ${ }^{\circ}$ 2100 sensor will be referred to as 'Palas system'.

Figure 2

\subsubsection{PM 2.5 cyclone}

In addition to the two optical sensors described above, a traditional PM2.5 cyclone (Fig. 3) was used to test the same aerosol in the same setting to serve as a reference device, which is based on a gravimetrical measuring principle. This reference is free of assumptions about the physical properties of the measured particles and can provide a true PM2.5 concentration for the duration of the experiment. The cyclone used in this experiment is a sharp cut cyclone of type BGI SCC designed in a way to match the separation of the USEPA PM2.5 reference device Well Impactor Ninety-Six [28] at a volumetric flowrate of $16.67 \mathrm{I} \mathrm{min}^{-1}$. The PM2.5-relevant part of the particles pass the cyclone and is subsequently collected by an absolute filter (high efficiency glass fiber filter), allowing the PM2.5 concentration of the gas for the whole duration of the experiment to be determined, indicating if the results provided by the digital sensors lie within the correct magnitude.

Figure 3

\subsection{Test Dust}

The low-cost PM sensor was tested using PURAL SB (Charge 145601, $x_{50}=45 \mu \mathrm{m}$, Sasol [29]) as test aerosol. As shown in Fig. 4. it is a fine, white powder and suitable for this experiment as it is chemically inert, easy to handle and has no tendencies to form agglomerations, i.e. has good dispersion properties. The dust has a density of $2.8 \mathrm{~g} \mathrm{~cm}^{-3}$, a loose bulk density of $600-850 \mathrm{~g} \mathrm{l}^{-1}$ and a packed bulk density of 800 to $1100 \mathrm{~g} \mathrm{I}^{-1}$. In this experiment the clean-gas side was investigated, therefore particle size distributions in this study differ from the raw material.

Figure 4

\subsection{Filter Medium}

Low-cost sensor and reference devices are compared in their performance quantifying the emissions of a filter medium with a diameter of $15 \mathrm{~cm}$. The filter medium is a fine dust filter (Heimbach 866E75) characterized by its very fine fibers ( $1.0 \mathrm{dtex}$ ). Having undergone 5000 regeneration cycles, the medium used in this study is well aged for minimal emissions and stable filter cake generation [30].

\subsection{Experimental arrangement and conditions}

Experiments are conducted with a modified VDI Type 1 filter-testing unit [31, ISO 11057] (see Fig. 5a). The test dust is dispersed and flows in a stream of compressed clean air through the raw-gas column. Part of the gas stream, including the penetrating particles, enters the measurement chamber through the test filter. The flowrate entering the measuring chamber is controlled by a mass flow controller and pump. The measurements of the welas 2100 and the SDS011 are taken simultaneously. A test flowrate enters an isokinetic probe, which is connected to the welas 2100 . The SDS011 is directly built into the measuring chamber and placed next to the isokinetic probe, precisely aligned as shown in Fig. $5 \mathrm{a}$ and Fig. $5 \mathrm{~b}$ to prevent any slipstream effects. To obtain reference values for the optical devices a PM2.5 cyclone is connected to the measuring chamber, removing all particles out of a flow of $1 \mathrm{~m}^{3} \mathrm{~h}^{-1}$ according to the specified flow rate of the PM2.5 cyclone. During surface filtration, a filter cake is building up on the raw-gas side of the filter, which increases the separation of aerosol from the gas stream, which in turn leads to lower particle 
concentrations in the measuring chamber. With the growth of the filter cake, the pressure drop over the filter increases. The regeneration of the test filter in this experiment is initiated through a compressed air impulse that blows against the filter in opposite direction of the raw-gas flow and pushes off most of the filter cake to reduce the pressure drop. In this study, the filter regeneration was initiated at a set combined pressure drop of $1000 \mathrm{~Pa}$ across filter medium and cake. Experiments were conducted at $21{ }^{\circ} \mathrm{C}$ ambient air temperature and between $40 \%$ and $50 \%$ relative humidity, with a duration of 457 minutes and a total number of 58 regeneration cycles.

Figure 5 (a), (b)

\section{Results and Discussion}

The performance of the low-cost PM sensor SDS011 was evaluated measuring PM2.5 concentrations of PURAL ${ }^{\circledR}$ SB emissions of a surface filter. Collected data were compared to the results of the Palas system (see Sect. 2.2.1) and a PM2.5 cyclone.

Particles passing through the test filter into the measuring chamber are predominantly smaller than $2 \mu \mathrm{m}$ with detailed size distributions shown in Fig. 6a and Fig. 6b, respectively.

Figure 6 (a), (b)

\subsection{Emission measurements of SDS011 and Promo 2000}

Comparing the two sensors with respect to the progression of their PM signal along the time axis, it can be said that the SDS011's reaction to the changes in the particle concentration within the system shows a similar trend to that of the Palas system. The SDS011 reacted instantaneously to concentration increase in the measuring chamber, caused by the regeneration of the filter at a pressure drop of $1000 \mathrm{~Pa}$, leading to an increased amount of particles passing through before a new filter cake is built (see Fig. 7).

Figure 7

Fig. 8 and Fig. 9 show the recorded PM2.5 and PM10 values respectively. The PM2.5 and PM10 values recorded by the Palas system show a difference in an order of magnitude compared to the values recorded by the low-cost PM sensor. The local maxima for the SDS011 remain just below 200 $\mu \mathrm{g} \mathrm{m}{ }^{-3}$ for the PM2.5 recording, which is well below the reported detection limit of $999.9 \mu \mathrm{g} \mathrm{m}^{-3}$. It is noticeable that although both sensors report one value per second, the SDS011 curve is smoothened and does not present temporary concentration increases in the measuring chamber. The data presented for the Palas system is clearly more fluctuating according to concentration changes following the filter regeneration. Therefore, for a true quantitative comparison between the two sensors it would be necessary to integrate the measurement signals over defined time periods.

Figure 8

Figure 9

\subsection{Comparison of optical results to gravimetrical result}

The cyclone was implemented as gravimetrical reference device to determine the overall PM2.5 value. The particles collected on the absolute filter behind the PM2.5 cyclone were converted into a PM2.5 concentration for the total analyzed volume over the total duration of the experiment. Subsequently comparing this value to the average PM2.5 concentration determined by the Palas ${ }^{\circ}$ system and the SDS011 provides information about the accuracy of the optical measuring devices.

To ensure the accuracy of the PM2.5 value for the Palas system for the dust used in this study, a calibration curve between optical diameter and aerodynamic diameter of the test dust was produced using an Aerodynamic Particle Sizer spectrometer 3321 by TSI ${ }^{\circ}$. The optical diameter measured by 
the Palas system was thereby converted into an aerodynamic diameter. Including the density of PURAL ${ }^{\circ}$ SB (see Sect. 2.3) the PM2.5 weighting factor for this dust was calculated with an according calibration curve. This conversion method leads to a result within an accuracy of $20 \%$ [32]. The concentration obtained by the cyclone is $69 \mu \mathrm{g} \mathrm{m}^{-3}$, while the concentrations determined by the optical devices are lower, $66 \mu \mathrm{g} \mathrm{m}^{-3}$ and $44 \mu \mathrm{g} \mathrm{m}^{-3}$ for the Palas system device and for the SDS011 low cost device, respectively (see Fig. 10). The concentration obtained for the Palas system lies within the expected accuracy and thereby validates our reference device.

Figure 10

\subsection{Limitations and potential}

The recordings of the SDS011 show that its application comes with certain limitations. Although its signal response reacts timely to a fast increase in concentration, the relaxation of the signal is delayed (Fig. 8, Fig. 9). The quantitative comparison between the SDS011, the Palas system and the cyclone showed, that the low-cost sensor records emissions, which are well below the recorded values of the reference devices. The low-cost sensor is supplied with an unknown calibration not intended for emission control, but with a suitable calibration for this purpose, using particles in the desired target application, a quantitative comparison between the devices could lead to better results in the future. Qualitatively, the SDS011 could describe the system during the experiments accurately and in an affordable, easily implemented way. These low-cost PM sensors show particular potential for applications and emission measurements where multiple sensors are required and system parameters such as temperature and humidity are controlled. In the future they could also be implemented with additional control systems for other system parameters, while still leading to overall cost savings if no expensive professional device is needed for measuring particle concentrations. But a true cost calculation cannot be provided at this point. Previously, only capital costs of low-cost sensors have been considered. A true cost evaluation can only be carried out, after a suitable calibration method has been found and maintenance costs, such as cleaning, upkeeping and periodical verification of the calibration, can be taken into account. Maintenance costs are likely to make the largest contribution to the overall costs and could potentially be the main drawback of this method.

Next, multiple SDS011 sensors and comparable low-cost PM sensors will be implemented in a multiple bag pilot filter plant to measure the emission contribution of each filter individually. Further experiments in the emission control will be carried out in order to investigate the suitability and limitations of low-cost sensors.

\section{Conclusion and Outlook}

The performance of the commercial SDS011 low-cost sensor was evaluated measuring the emissions of a surface filter. A welas 2100 sensor with a Promo 2000 aerosol spectrometer by Palas ${ }^{\circ}$ was used as a reference instrument. These first emission control experiments with the SDS011 low-cost sensor show qualitatively the same trend as the recordings of the Palas system. Particle concentration changes in the measuring chamber were recorded promptly. Note that it was ensured that the concentrations used in this experiment were well below the detection limit of $999.9 \mu \mathrm{g} \mathrm{m}^{-3}$ of the SDS011, which only holds for very efficient filter media during emission experiments of this scale and is one of the drawbacks of using low-cost sensors in this setting.

The potential of low-cost sensors is high in the application of emission measurements as system parameters such as humidity and temperature can be controlled during this application and subsequently do not interfere with the sensor's measurements. Additionally, the low capital investment of these sensors holds great potential for research applications that require qualitative system descriptions or comparison between multiple emission sources in one system, leading to an instantaneous high special resolution.

This article is protected by copyright. All rights reserved. 


\section{References}

[1] S. Wu, Y. Ni, H. Li, L. Pan, D. Yang, A. Baccarelli, F. Deng, Y. Chen, M. Shima, X. Guo, Environ. Int. 2016, 94, 76-82. DOI: 10.1016/j.envint.2016.05.004

[2] M. Pascal, M. Corso, O. Chanel, C. Declercq, C. Badaloni, G. Cesaroni, S. Henschel, K. Meister, D. Haluza, P. Martin-Olmedo, S. Medina, Aphekom group, Sci. Total Environ. 2013, 4449, 390-400, DOI: 10.1016/j.scitotenv.2013.01.077

[3] H. Li, J. Cai, R. Chen, Z. Zhao, Z. Ying, L. Wang, J. Chen, K. Hao, P. L. Kinney, H. Chen, H. Kann, Circulation 2017, 136 (7), 618-627. DOI: 10.1161/CIRCULATIONAHA.116.026796

[4] M. Bentayeb, V. Wagner, M. Stempfelet, M. Zins, M. Goldberg, M. Pascal, S. Larrieu, P. Beaudeau, S. Cassadou, D. Eilstein, L. Filleul, A. Tertre, S. Medina, L. Pascal, H. Prouvost, P. Quénel, A. Zeghnoun, A. Lefranc, Environ. Int. 2015, 85, 5-14. DIO: 10.1016/j.envint.2015.08.006

[5] J. Fan, S. Li, C. Fan, Z. Bai, K. Yang, Environ. Sci. Pollut. Res. Int. 2016, 23 (1), 843-50. DOI: 10.1007/s11356-015-5321-x

[6] O. Raaschou-Nielsen, R. Beelen, M. Wang, G. Hoek, Z. J. Andersen, B. Hoffmann, M. Stafoggia, E. Samoli, G. Weinmayr, K. Dimakopoulou, M. Nieuwenhuijsen, W. W. Xun, P.

[7] A. M. Kaapen, P. J. Borm, C. Albrecht, R. P. Schins, Int. J. Cancer 2004, DOI: 10.1002/ijc.11708

[8] www.everyaware.eu (Accessed on October 25, 2017)

[9] www.opensensemap.org (Accessed on October 25, 2017)

[10] www.citi-sense.eu (Accessed on October 25, 2017)

[11] www.aircasting.org (Accessed on October 25, 2017)

[12] www.inovafitness.com (Accessed on Oktober 25, 2017

[13] www.luftdaten.info (Accessed on October 25, 2017)

[14] C. Borrego, A. Costa, J. Ginja, M. Amorim, M. Coutinho, K Karatzas, Th. Sioumis, N. Katsifarakis, K. Konstantinidis, S. De Vito, E. Esposito, P. Smith, N. André, P. Gérard, L.A. Drancis, N. Castell, P. Schneider, M. Viana, M. C. Minguillón, W. Reimringer, R. P. Otjes, O. von Sicard, R. Pohle, B. Elen, D. Suriano, V. Pfister, M. Prato, S. Dipinto, M. Penza, Atmos. Environ. 2016, 147, 246-263. DOI: 10.1016/j.atmosenv.2016.09.050

[15] S. Castellini, B. Moroni, D. Cappelletti, Measurement 2014, 49, 99-106. DOI: 10.1016/j.measurement.2013.11.045

[16] M. Viana, I. Rivas, C. Reche, A. S. Fonseca, N. Pérez, X. Querol, A. Alastuey, M. ÁlvarezPedrerol, J. Sumyer, Atmos. Environ. 2015, 123, 220-228. DOI: 10.1016/j.envres.2015.05.017

[17] S. Sousan, K. Koehler, L. Hallett, T. M. Peters, Aerosol Sci. Technol. 2016, 50, 1352-1365. DOI: 10.1080/02786826.2016.1232859

[18] N. Castell, F. R. Dauge, P. Schneider, M. Vogt, U. Lerner, B. Fishbain, D. Broday, A. Bartonova, Environ. Int. 2017, 99, 293-302. DOI: 10.1016/j.envint. 2016.12.007

[19] D. Liu, Q. Zhang, J. Jiang, D. Chen, J. Aerosol Sci. 2017, 112, 1-10. DOI: 10.1016/j.jaerosci.2017.05.011

[20] LUBW Landesanstalt für Umwelt, Messungen und Naturschutz Baden-Württemberg (Juni 2017) Messungen mit dem Feinstaubsensor SDS011, Retrieved from http://www4.lubw.badenwuerttemberg.de/servlet/is/268831/messungen_mit_dem_feinstaubsensor_sds011.pdf?command= downloadContent\&filename=messungen_mit_dem_feinstaubsensor_sds011.pdf

This article is protected by copyright. All rights reserved. 
[21] L. R. Crilley, M. Shaw, R. Pound, L. J. Kramer, R. Price, S. Young, A. C. Lewis, F. D. Pope, Atmos. Meas. Tech. 2017. DOI: 10.5194/amt-2017-308

[22] A. C. Lewis, J. D. Lee, P. M. Edwards, M. D. Shaw, M. J. Evans, S. J. Moller, K. R. Smith, J. W. Buckley, M. Ellis, S. R. Gillot, A. White, Faraday Discuss. 2016, 189, 85-103. DOI: 10.1039/c5fd00201j

[23] K. R. Smith, P. Edwards, M. J. Evans, J. D. Lee, M. D. Shaw, F. A. Squires, A. Lewis, Faraday Discuss. 2017, 200, 621-637. DOI: 10.1039/c7fd00020k

[24] N. Streibl, Researchgate 2017, DOI: 10.13140/RG.2.2.21095.75683

[25] Manikonda, N. Zíková, P. K. Hopke, A. R. Ferro, J. Aerosol Sci. 2016, 102, 29-40. DOI: 10.1080/02786826.2015.1100710

[26] www.watterott.com/de/Nova-SDS011-Feinstaub-Sensor (Accessed on October 25, 2017)

[27] www.palas.de/de/product/promo2000 (Accessed on October 25, 2017)

[28] FRL-9966-90-ORD, Ambient air monitoring reference and equivalent methods, USEPA United States Environmental Protection Agency, 1997.

[29] http://www.sasolgermany.de/fileadmin/doc/alumina/Neu_2017/0372.SAS-BRInorganics_Pural_Catapal_WEB.pdf (Accessed on May 28, 2018)

[30] S. Sobich, FILTECH 2018, Cologne, March 2018.

[31] P. Gäng, F. Löffler, Proc. 9th World Clean Air Congress, Montreal, August 1992.

[32] J. Binnig, Ph.D. Thesis, Karlsruher Institut für Technologie 2011. 
Figure legends

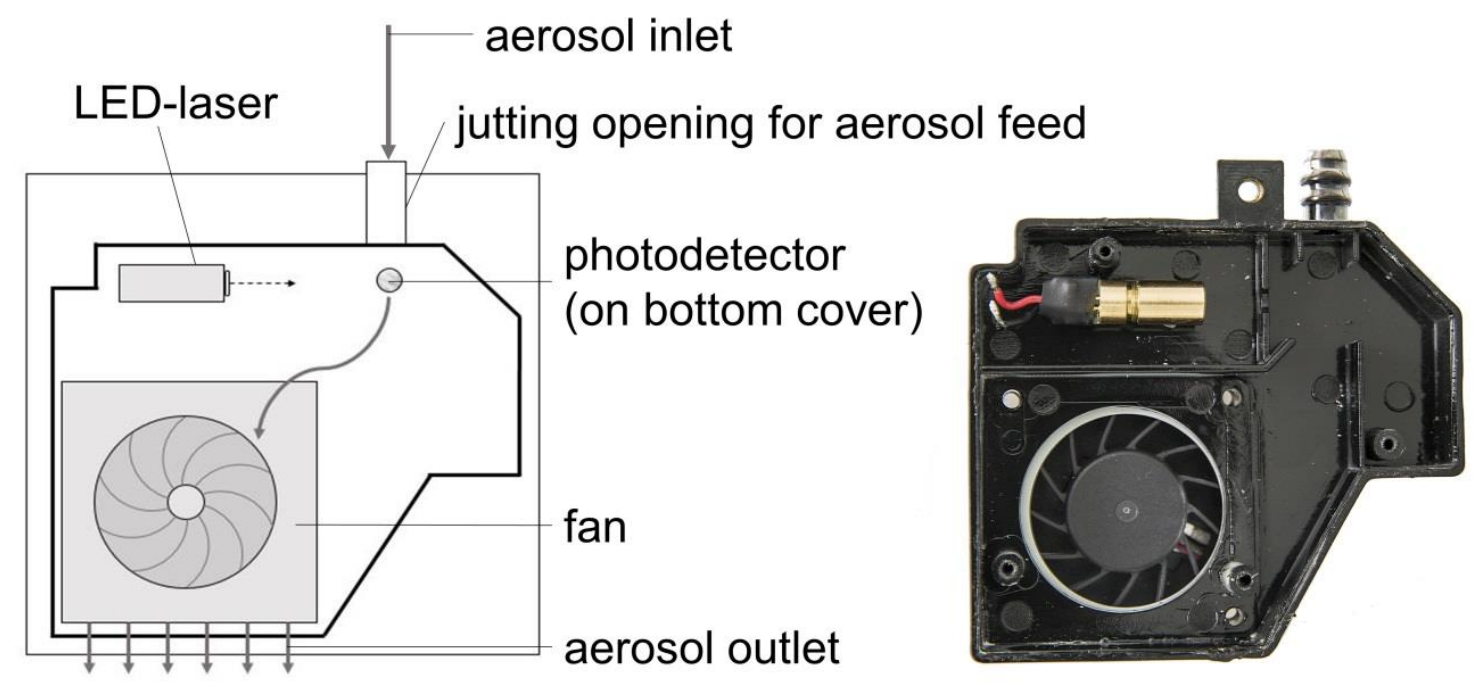

Figure 1. Schematic diagram and picture of internal configuration of NOVA SDS011 low-cost sensor

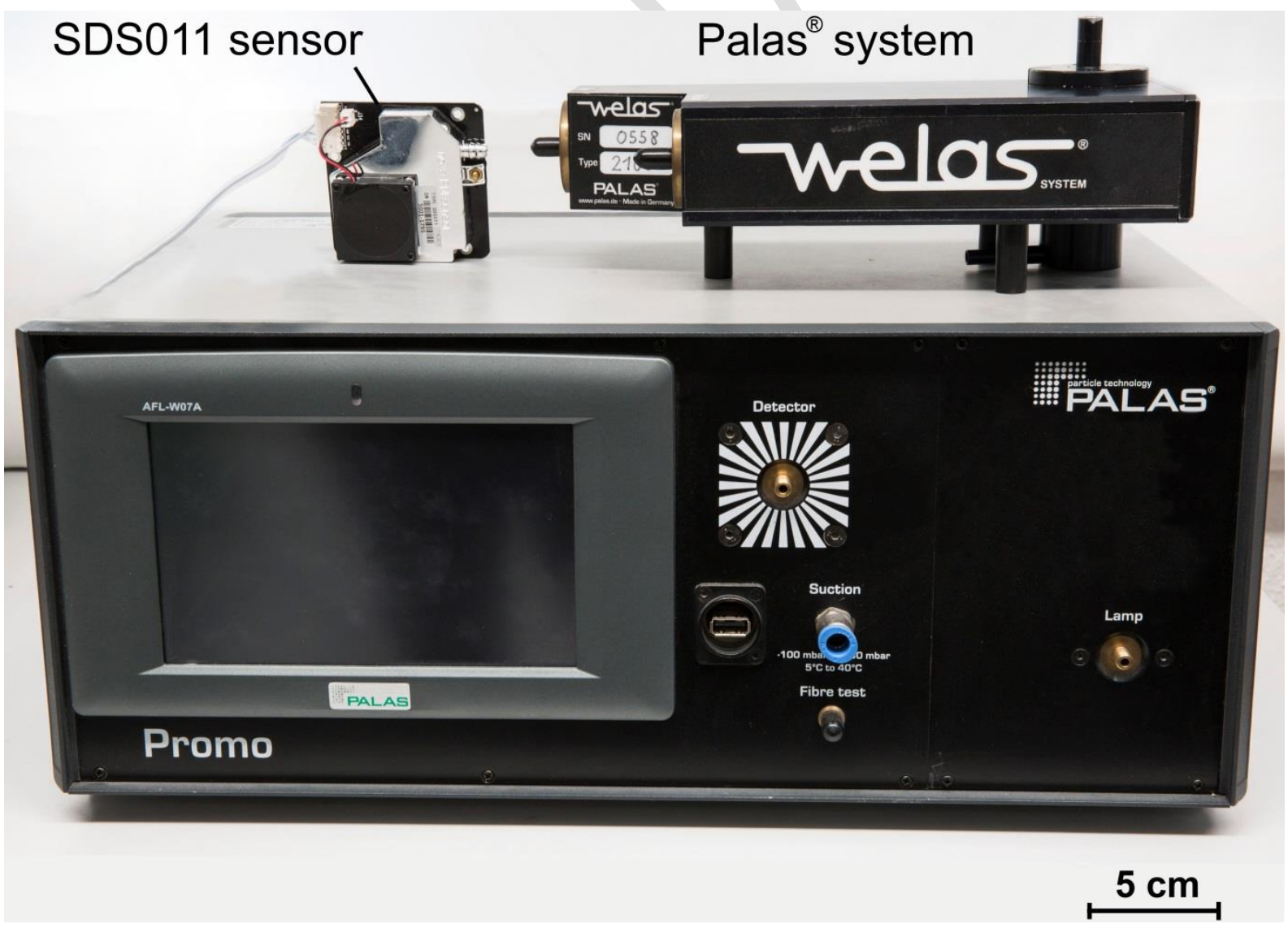

Figure 2. Picture of Promo 2000 unit with welas 2100 sensor and SDS011 low-cost sensor

This article is protected by copyright. All rights reserved. 


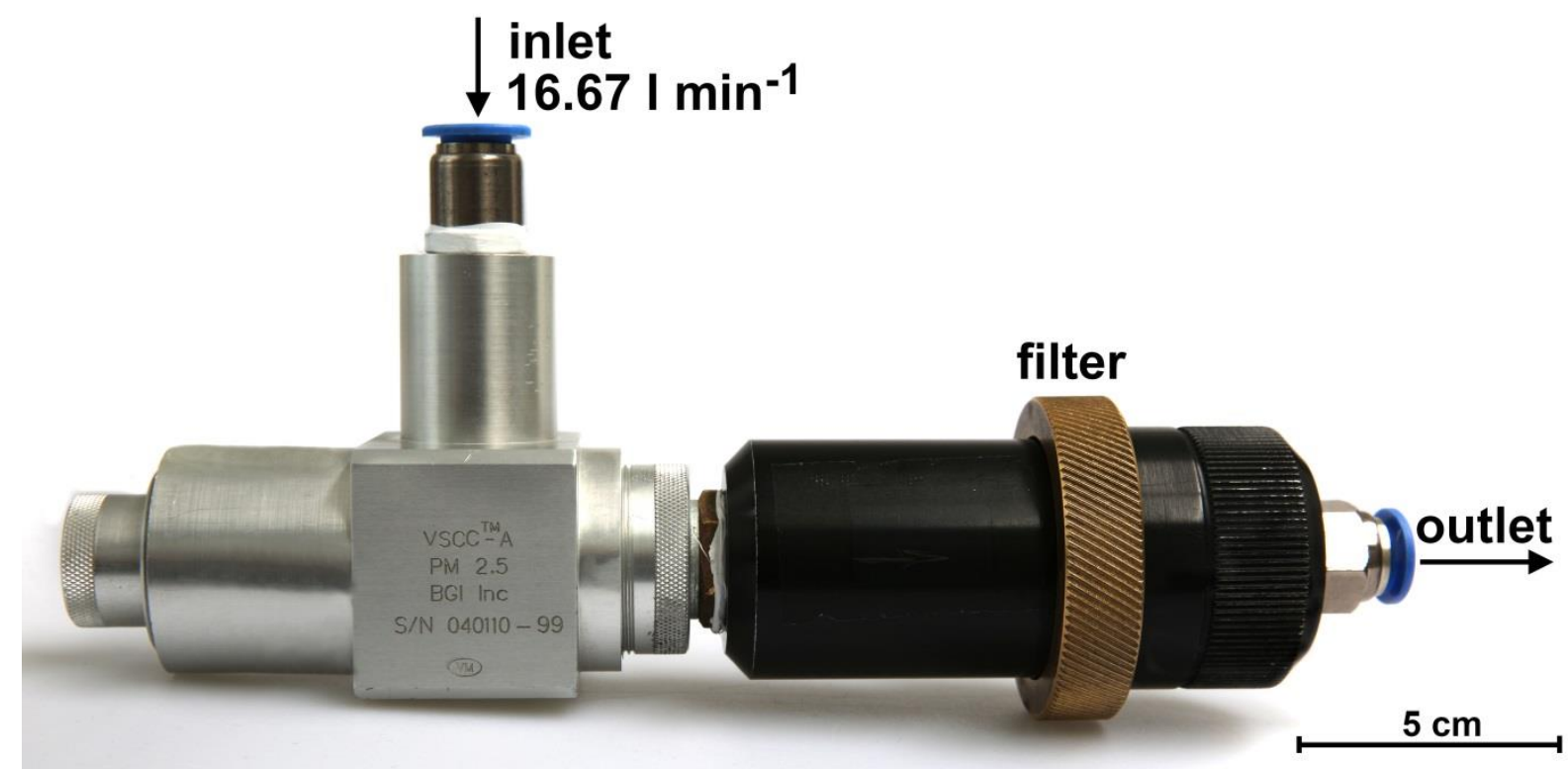

Figure 3. Picture of the cyclone used in this study, indicating the flow of gas and particles and the location of the absolute filter

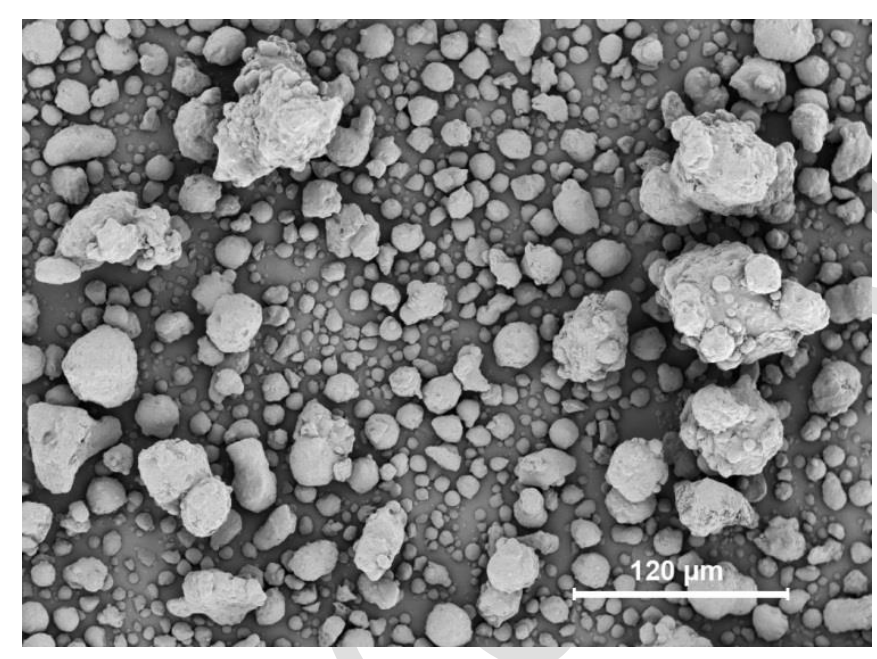

Figure 4. Picture of test dust PURAL ${ }^{\oplus}$ SB on test filter taken with scanning electron microscope 

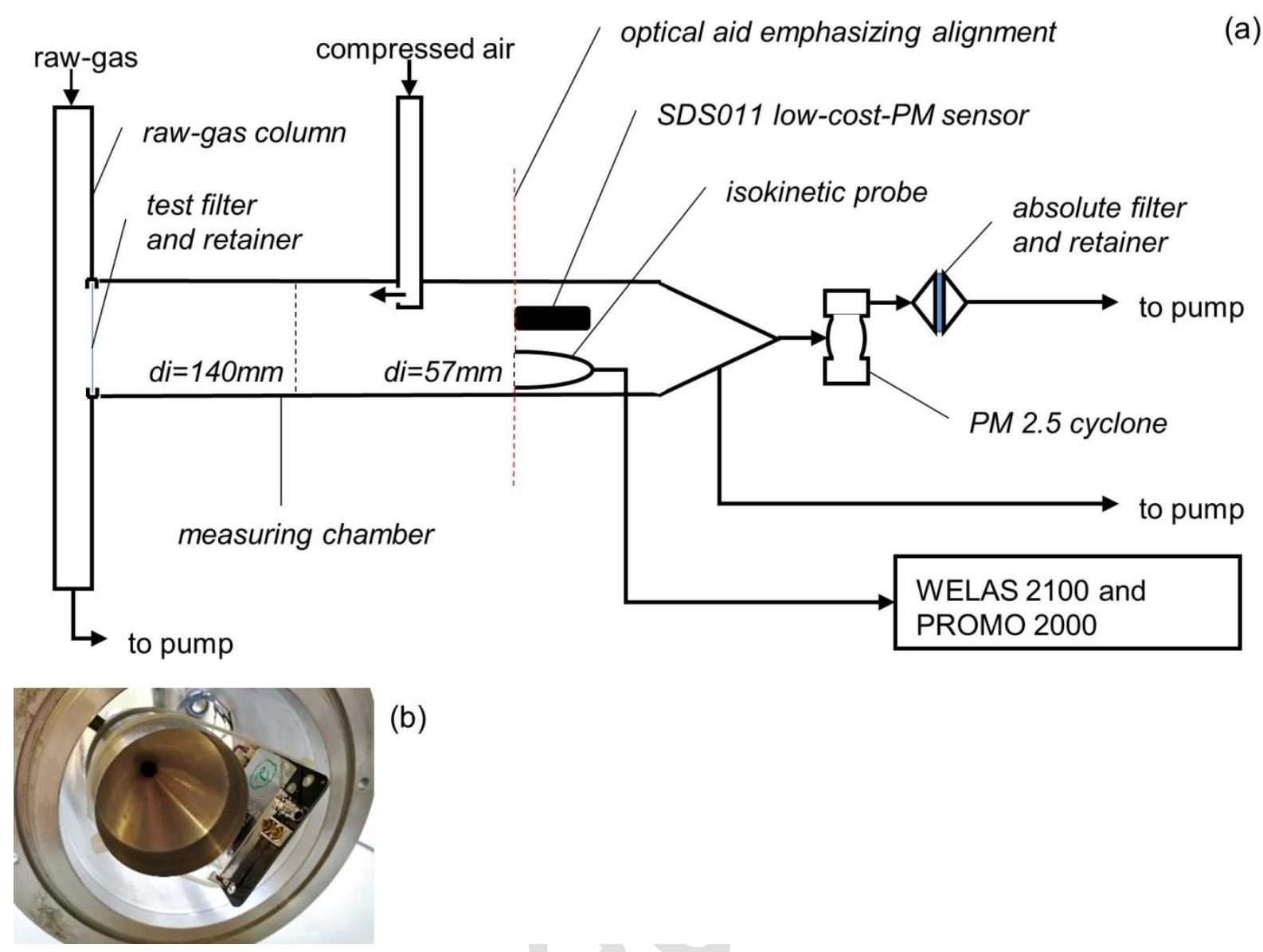

(b)

Figure 5. (a) Schematic diagram of experimental setup including (b) a picture of the SDS011 sensor and the isokinetic probe connected to the welas 2100 built into the measuring chamber 

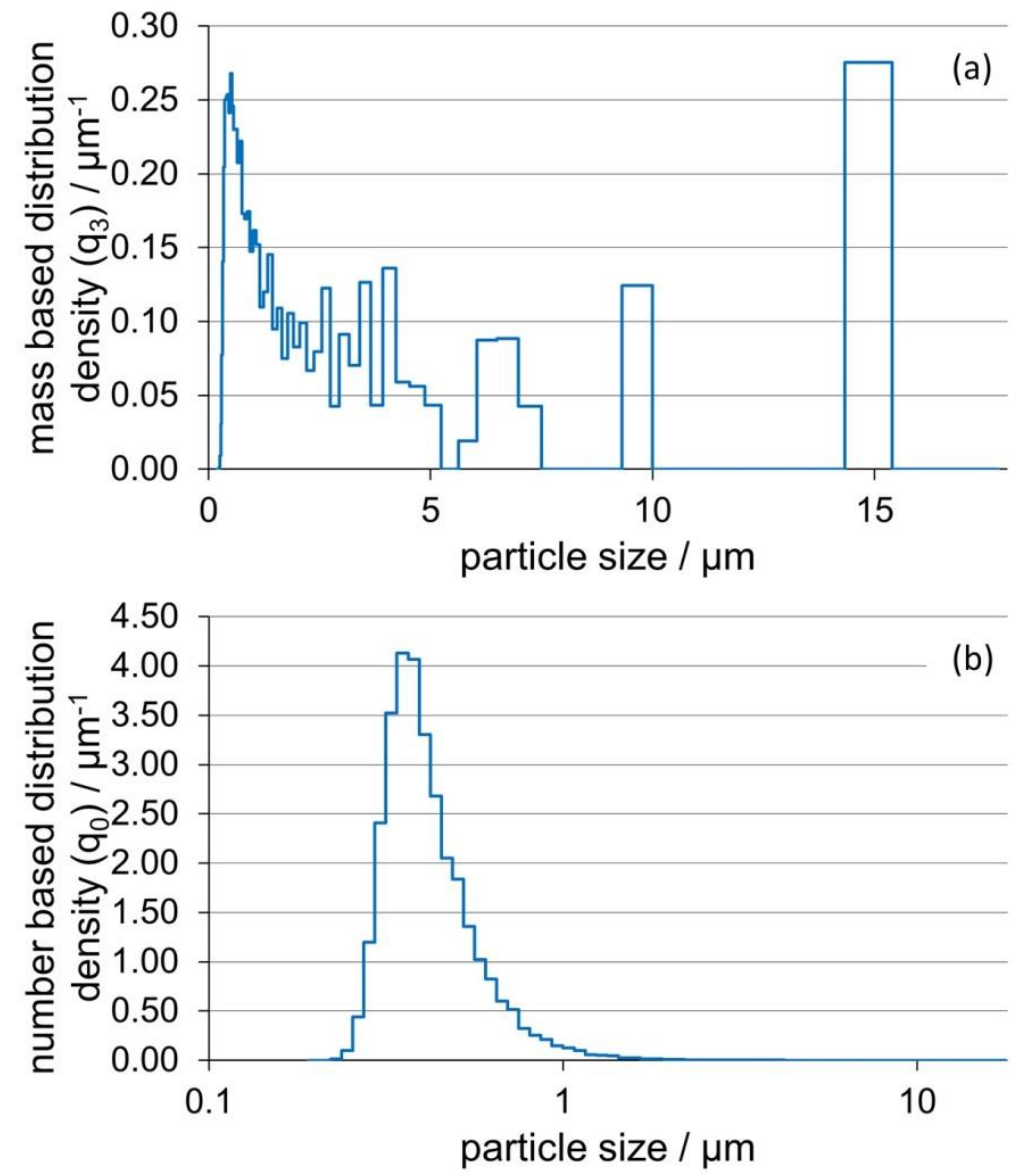

Figure 6. Particle size distribution densities (a) mass based and (b) number based of the test dust PURAL ${ }^{\circ} \mathrm{SB}$ in the measuring chamber as recorded by the Palas system

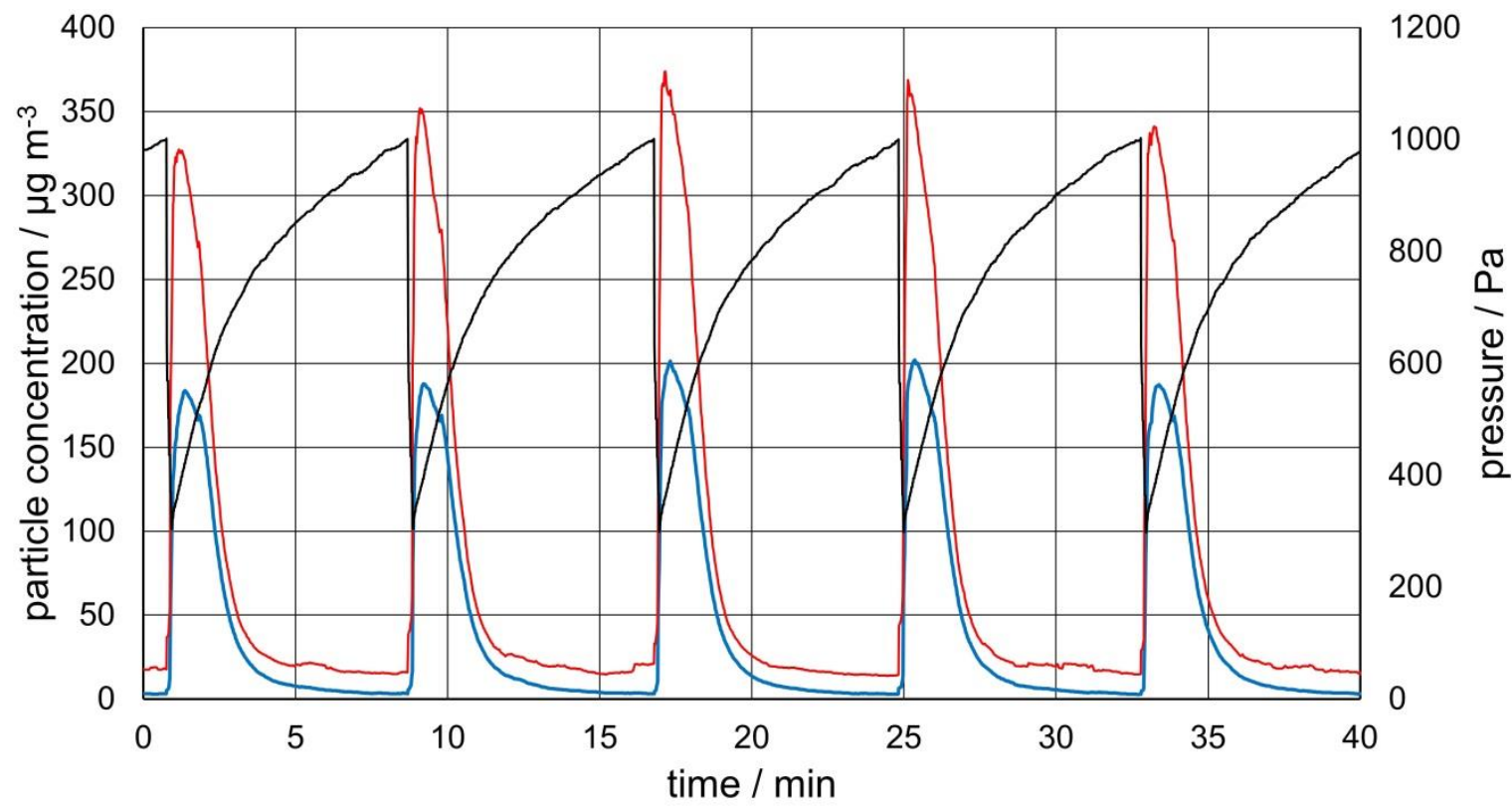

-PM 2.5 -PM 10 -Pressuredrop over test filter

Figure 7. Comparison of SDS011 PM2.5 and PM10 results with the pressure drop increase over the filter and the according filter regeneration at $1000 \mathrm{~Pa}$

This article is protected by copyright. All rights reserved. 


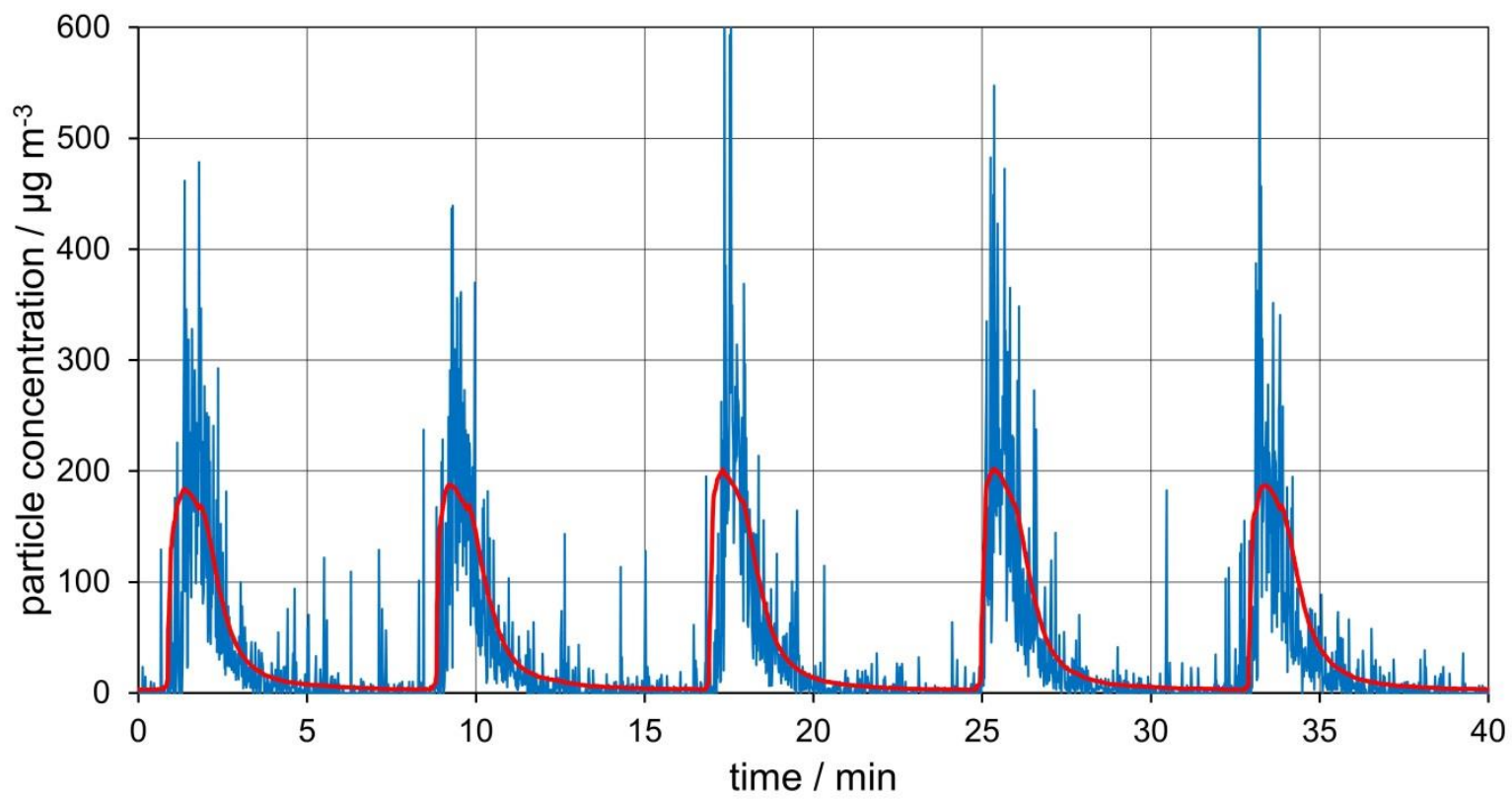

-Palas ${ }^{\circledR}$ system -SDS011

Figure 8. PM2.5 concentrations for SDSO11 and Palas system

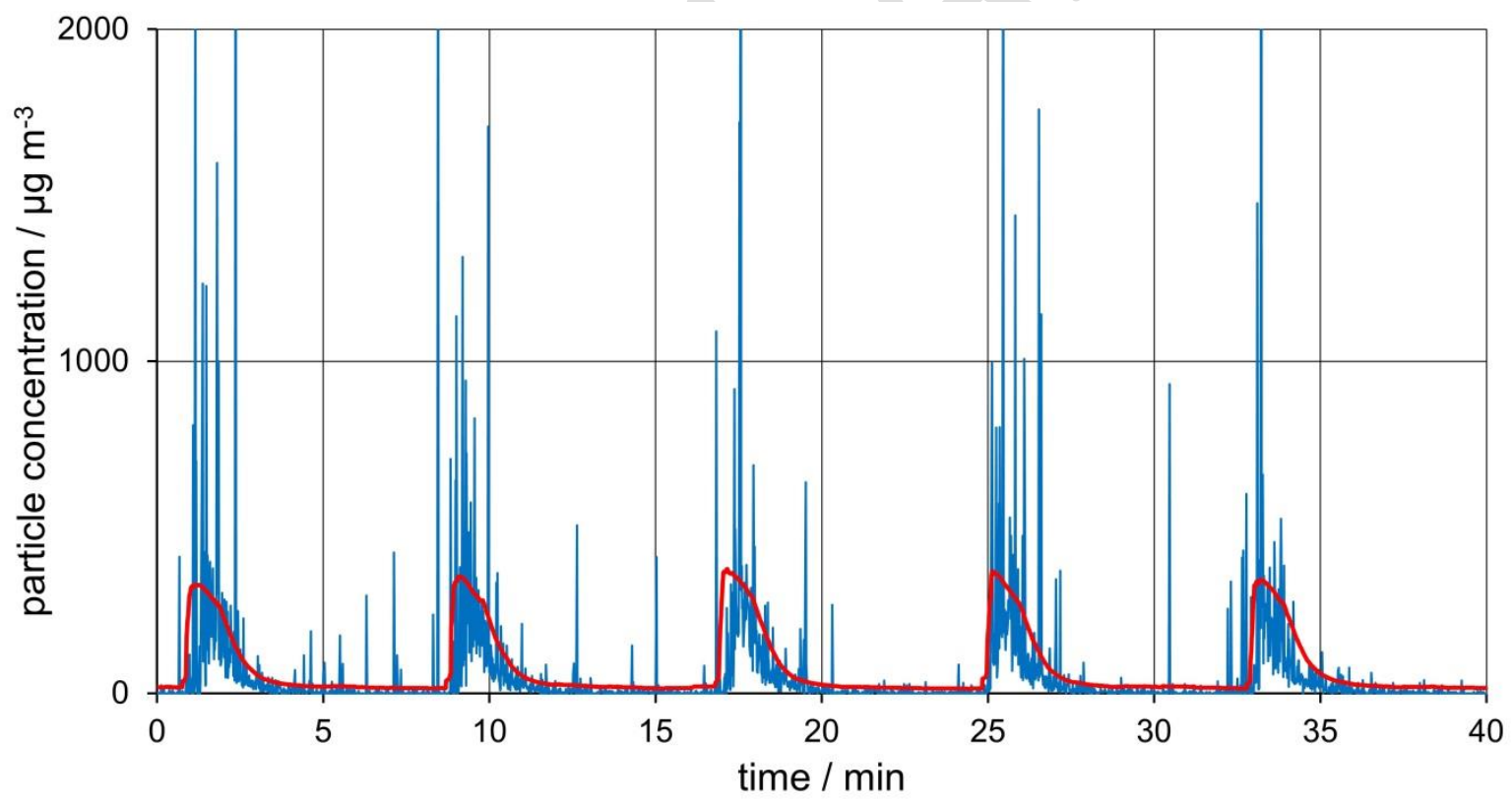

-Palas ${ }^{\circledR}$ system -SDS011

Figure 9. PM10 concentrations of SDS011 and Palas system 


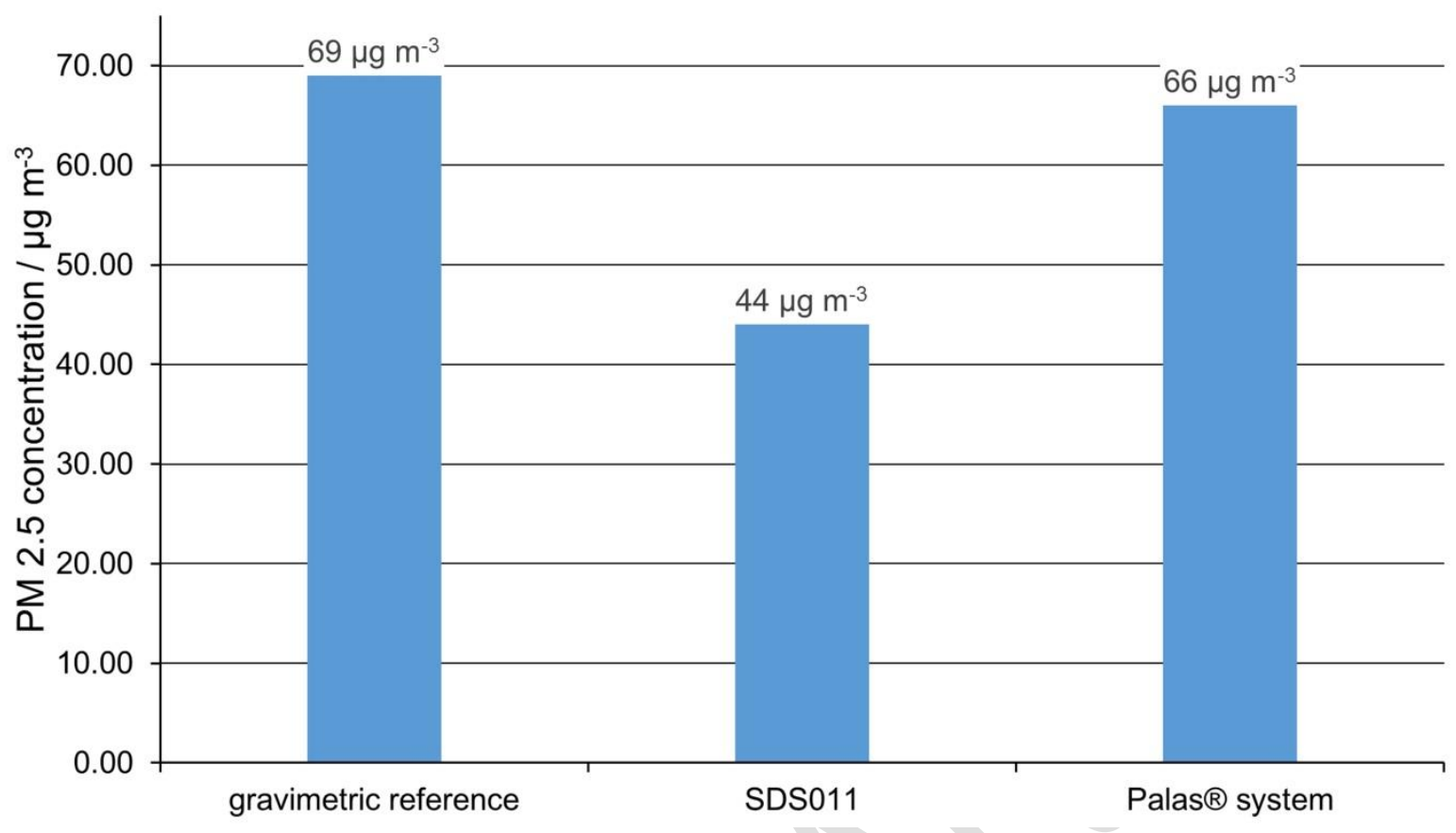

Figure 10. PM2.5 values for the whole duration of the experiment for all three measuring devices (from left to right): Cyclone, SDS011 low-cost sensor, Palas system. 


\section{Tables and table legends}

Table 1. Specification of sensors used in this study

\begin{tabular}{|c|c|c|c|}
\hline Type & Low-cost sensor & $\begin{array}{l}\text { Sensor for professional } \\
\text { purpose }\end{array}$ & Cyclone \\
\hline Provider & NOVA & PALAS & Mesa Labs \\
\hline Model \# & SDS011 & $\begin{array}{l}\text { PROMO 2000, } \\
\text { welas } 2100 \text { sensor }\end{array}$ & BGI SCC \\
\hline Size & $71 \times 70 \times 23 \mathrm{~mm}$ & $\begin{array}{l}185 \times 450 \times 315 \mathrm{~mm}, 50 \\
\times 250 \times 100 \mathrm{~mm}\end{array}$ & $110 \times 250 \times 60 \mathrm{~mm}$ \\
\hline Weight (approx.) & $0.045 \mathrm{~kg}$ & $8 \mathrm{~kg}+2.8 \mathrm{~kg}$ & $1.195 \mathrm{~kg}$ \\
\hline Measurements & PM2.5, PM10 & $\begin{array}{l}\text { Mass concentrations } \\
\text { (size resolved), } \\
\text { Conversion to PM1, } \\
\text { PM2.5, PM10 }\end{array}$ & PM2.5 \\
\hline Sampling method & Fan & Pump & Pump \\
\hline Flow rate & $0.51 \mathrm{~min}^{-1}$ & $5.01 \mathrm{~min}^{-1}$ & $16.67 / \mathrm{min}^{-1}$ \\
\hline Detection Principle & Optical & Optical & Gravimetrical \\
\hline Detectable size range & $0.3-10 \mu \mathrm{m}$ & $0.3-17 \mu \mathrm{m}$ & PM2.5 \\
\hline Concentration range & $0.0-999.9 \mu \mathrm{g} \mathrm{m}^{-3}$ & $0-5 \times 10^{5}$ particles $\mathrm{cm}^{-3}$ & NA \\
\hline Response time & $<10 \mathrm{~s}$ & $<1$ s & NA \\
\hline Max. relative error & $\pm 15 \%, \pm 10 \mu \mathrm{g} \mathrm{m}^{-3}$ & NA & NA \\
\hline Cost (approx.) & 30 Euro & 30,000 Euro & NA \\
\hline
\end{tabular}




\section{Short text for the table of contents section}

An innovative approach utilizing low-cost particulate matter sensors for emission control is introduced. Applicability and new limitations are tested in a laboratory setting measuring particulate emissions of surface filters and comparing results with conventional measuring devices. 